\title{
BF3.SiO2: an efficient catalyst for the synthesis of azo dyes at room temperature
}

\author{
Bi Bi Fatemeh Mirjalili ${ }^{a^{*}}$, Abdolhamid Bamoniri ${ }^{\mathbf{b}}$ and Ali Akbari ${ }^{\mathrm{a}}$
}

${ }^{a}$ Department of Chemistry, College of Science, Yazd University, Yazd, P.O.Box 89158-13149, I. R. Iran

${ }^{b}$ Department of Organic Chemistry, Faculty of Chemistry, University of Kashan, Kashan, I.R.Iran

\begin{tabular}{|c|c|}
\hline ART ICLE I NFO & ABSTRACT \\
\hline $\begin{array}{l}\text { Article history: } \\
\text { Received May 30, } 2012 \\
\text { Received in Revised form } \\
\text { June 2, } 2012 \\
\text { Accepted } 24 \text { June } 2012\end{array}$ & $\begin{array}{l}\text { A rapid one-pot method has been developed for the synthesis of azo dyes via sequential } \\
\text { diazotization-diazo coupling of aromatic amines with coupling agents at room temperature in } \\
\text { the presence of } \mathrm{BF} 3 . \mathrm{SiO} 2 \text { as acidic catalyst. The obtained aryl diazonium salts bearing silica } \\
\text { supported boron tri-flouride counter ion was sufficiently stable to be kept at room temperature } \\
\text { in the dry state. }\end{array}$ \\
\hline
\end{tabular}

able online

24 June 2012

Keywords:

Azo dye

$\mathrm{BF}_{3} \cdot \mathrm{SiO}_{2}$

Stable diazonium salts

Diazotization

Lewis acid

\section{Introduction}

Azo compounds are the most widely class of industrial synthesized organic dyes due to their versatile application in various fields, such as dyeing textile fiber ${ }^{1}$, biological-pharmacological activities $^{2}$. Azo dyes have been synthesized via coupling of diazotized aromatic amines with activated aromatic compounds. Previously, aromatic amines have been converted to diazonium salts in the presence of $\mathrm{NaNO}_{2}$ and a mineral acid at $0-5^{\circ} \mathrm{C}$.

Boron tri-flouride.etherated $\left(\mathrm{BF}_{3} \cdot \mathrm{OEt}_{2}\right)$, as thin, coloured and fuming liquid, is used in industry and organic synthesis. Since, $\mathrm{BF}_{3} \cdot \mathrm{OEt}_{2}$ is liquid that fumes in air and reacts with the moisture to form $\mathrm{HF}$, the handling and the usability of it as liquid form is laborious and the supported form is indeed preferable. Silica supported boron tri-fluoride $\left(\mathrm{BF}_{3} \cdot \mathrm{SiO}_{2}\right)^{3-7}$ is a bench-top catalyst which is reusable,

* Corresponding author.

E-mail addresses: fmirjalili@gmail.com (B. B. Mirjalili) 
cheap, readily available, eco-friendly, versatile and efficient for promotion of many acid catalyzed organic reactions. This catalyst does not need special precautions for preparation, handling or storage, and it can be stored at ambient temperature for months without losing its catalytic activity.

In this article, we report a new protocol for synthesis of azo dyes in the presence of $37 \% \mathrm{BF}_{3} . \mathrm{SiO}_{2}$, at room temperature.

\section{Results and Discussion}

Due to the poor thermal stability of diazonium salts, they were previously synthesized around 0$10{ }^{\circ} \mathrm{C}$ and were handled below $0{ }^{\circ} \mathrm{C}$. In our investigation, the obtained aryl diazonium salts bearing silica supported boron tri-fluoride counter ion was sufficiently stable to be kept at room temperature in the dry state. In our new method, different kinds of aromatic amines, with electron-withdrawing groups as well as electron-donating groups, were rapidly converted to the corresponding azo dyes in the presence of $\mathrm{NaNO}_{2}$ and $37 \% \mathrm{BF}_{3} \cdot \mathrm{SiO}_{2}$ at room temperature (Scheme 1 and Table 1). Generally, a mixture of an aromatic amine $(2 \mathrm{mmol}), 37 \% \mathrm{BF}_{3} \cdot \mathrm{SiO}_{2}(0.2 \mathrm{~g})$ and sodium nitrite $(2 \mathrm{mmol})$ was ground in a mortar for 2-3 minutes at room temperature to obtain a homogeneous mixture. Then, 1 $\mathrm{mL}$ of water was gradually added to this mixture and mixed for $2 \mathrm{~min}$. Then, coupling agent ( $2 \mathrm{mmol})$ was added to the diazonium salt and ground for $2 \mathrm{~min}$. The whole process of diazotization and diazo coupling takes 6-7 minutes. The crude product was dissolved in chloroform and filtered to isolation of catalyst. The crude product was purified by re-crystallization in ethanol.

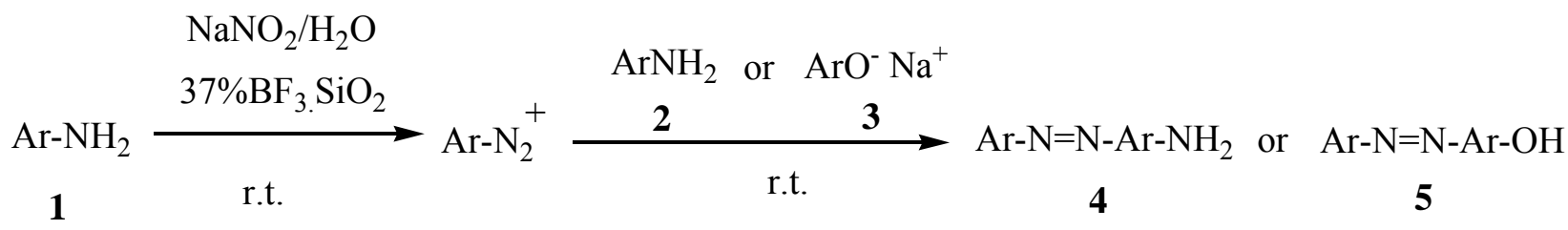

Scheme 1: Synthesis of azo dyes in the presence of silica supported boron tri-flouride

Table 1. Synthesis of azo dyes in the presence of $37 \% \mathrm{BF}_{3} \cdot \mathrm{SiO}_{2}$ at room temperature.

Entry $\mathrm{Amine} \mathrm{(1)}$


4<smiles>Nc1ccccc1</smiles>

5<smiles>Nc1ccc([N+](=O)[O-])cc1</smiles>

6

1d

7<smiles>CCc1ccc(N)cc1</smiles>

8

1f

9<smiles>Cc1ccc(Cl)cc1N</smiles>

10<smiles>CCc1ccc(N)cc1</smiles>

11<smiles>CC1([AlH2])CC(=O)C2=C(C1)Oc1ccc3ccccc3c1C2c1ccc(N)cc1</smiles>

$12 \mathbf{1 h}$

13

$1 a$

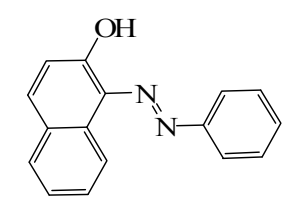

86

129-131

Sudan I

82

183-185

Para Red

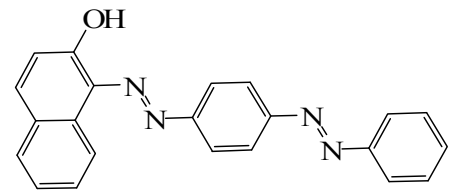

82

103-105

Sudan III

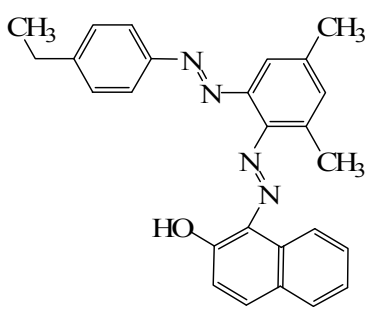

82

89-91

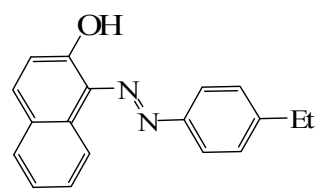

84

75-77

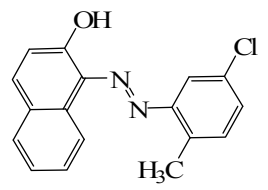

89

118-120

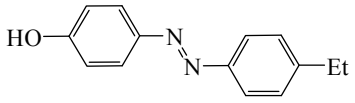

87

74-77

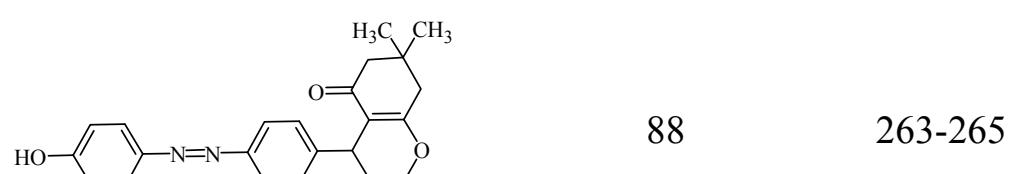

85

123-126

84

123-125 
14<smiles>Nc1ccc(C2c3c(ccc4ccccc34)OC3C=Cc4ccccc4C32)cc1</smiles>

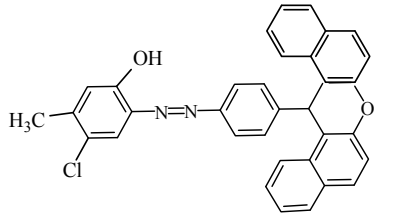

88

90-91

115-116

$15 \quad \mathbf{1 i}$

16

1i

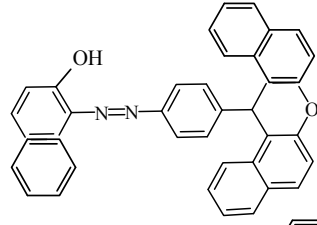

89

93
98-100

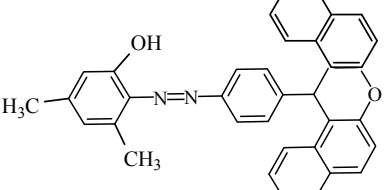

${ }^{\mathrm{a}}$ The ratio of amine (mmol): $\mathrm{NaNO}_{2}(\mathrm{mmol}): 37 \% \mathrm{BF}_{3} \cdot \mathrm{SiO}_{2}(\mathrm{~g})$ : coupling agent (mmol) is equal to 2:2:0.2:2

The amines $\mathbf{1 h}$, and $\mathbf{1 i}$ were obtained by reduction of corresponding nitro compounds $\mathbf{6}$ and 7 in the presence of $\mathrm{Na}_{2} \mathrm{~S} .9 \mathrm{H}_{2} \mathrm{O}$ and sulfur ${ }^{8}$ (Scheme 2). The substance 6 was synthesized via condensation of $\beta$-naphthol, dimedone and 4-nitrobenzaldehyde in the presence of $\mathrm{Bi}\left(\mathrm{NO}_{3}\right)_{3} \cdot \mathrm{SiO}_{2}$ and sodium dodecyl sulfate (SDS) in water at $40{ }^{\circ} \mathrm{C}$ (Scheme 3). The diarylxanthene 7 (Scheme 4) was synthesized via reaction between 4-nitrobenzaldehyde and 2-napththol in the presence of $37 \% \mathrm{BF}_{3} \cdot \mathrm{SiO}_{2}{ }^{3}$.

Ar- $\mathrm{NO}_{2} \underset{\mathrm{EtOH}, \mathrm{H}_{2} \mathrm{O}}{\stackrel{\mathrm{Na}_{2} \mathrm{~S} .9 \mathrm{H}_{2} \mathrm{O}, \mathrm{S}}{\longrightarrow}}$ Ar- $-\mathrm{NH}_{2}$

Scheme 2: reduction of aromatic nitro compounds

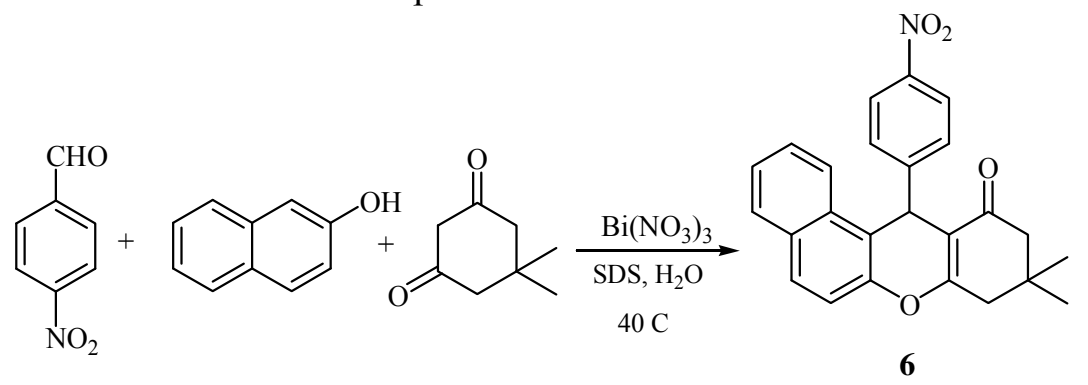

Scheme 3. Synthesis of 6 via condensation of $\beta$-naphthol, dimedone and 4-nitrobenzaldehyde<smiles>O=[N+]([O-])c1ccc(C2c3c(ccc4ccccc34)Oc3ccc4ccccc4c32)cc1</smiles>

Scheme 4. The structure of nitro diarylxanthene 7

\section{Conclusion}

We have demonstrated simple method for the synthesis of azo dyes using $\mathrm{BF}_{3} . \mathrm{SiO}_{2}$, as heterogeneous and efficient catalyst. Short reaction times, high yields, a clean process, simple methodology, easy work-up and green conditions are advantages of these protocols. 


\section{Acknowledgements}

The Central Iron Ore and Strategic of Research Centre gratefully acknowledged for the financial support for this work.

\section{Experimental}

\section{Materials and Methods}

All chemicals were purchased from Fluka and Merck companies. Products were characterized by IR, ${ }^{1} \mathrm{H}-\mathrm{NMR}$ and by comparison of their physical properties with those reported in the literature. IR spectra were run on a Bruker, Eqinox 55 spectrometer. ${ }^{1} \mathrm{H}$ NMR spectra were obtained using a Bruker Avans $400 \mathrm{MHz}$ spectrometer (DRX). Melting points were determined by a Buchi melting point B540 B.V.CHI apparatus.

Synthesis of 12-(4-nitrophenyl)-9, 9-dimethyl 8, 9, 10, 12-tetrahydrobenzo(a)xanthenes -11-one (6) in water

A mixture of 2-naphthol (1 mmol), 4-nitrobenzaldehyde (1 mmol), dimedone (1.2 mmol), $\mathrm{Bi}\left(\mathrm{NO}_{3}\right)_{3} \cdot \mathrm{SiO}_{2}(0.05 \mathrm{~g}), 0.01 \mathrm{~g}$ of sodium dodecyl sulfate (SDS) and $2 \mathrm{~mL}$ water was heated at $45^{\circ} \mathrm{C}$ in water bath. The progress of the reaction was monitored by TLC. After completion of the reaction, the mixture was poured into a separator funnel to isolate the product. The chloroform layer was evaporated carefully and the obtained solid was crystallized from ethanol : water $(80: 20)$ to afford the pure product.

Synthesis of azo dyes in the presence of $\mathrm{BF}_{3} \cdot \mathrm{SiO}_{2}$ at room temperature:

A mixture of $0.14 \mathrm{~g}(2 \mathrm{mmol})$ of $\mathrm{NaNO}_{2}$ and $0.2 \mathrm{~g} 37 \% \mathrm{BF}_{3} \cdot \mathrm{SiO}_{2}$ was grounded in a mortar. Then, 2 (mmol) of aromatic amine was added and grinded. After formation of diazonium salt, a solution containing $2 \mathrm{mmol}$ sodium phenoxide or naphthoxide was added and sufficiently grounded. The obtained azo dye was dissolved in ethanol and filtered to isolate of catalyst. The ethanol solution of azo dye was concentrated. By addition of water to ethanol solution, the azo dye was separated in pure solid form.

Physical and spectral data of some represented products

4-(2-(naphthalen-1-yl)diazen-1-yl)naphthalen-1-amine (Table 1, entry 1)

Dark brown, FT-IR: $v_{\max }(\mathrm{KBr})=3379,3481,1616,1462,1597,1462,1330,828,756 \mathrm{~cm}^{-1} .{ }^{1} \mathrm{H}$ $\operatorname{NMR}\left(\mathrm{CDCl}_{3}\right): 4.67\left(\mathrm{~s}, 2 \mathrm{H}, \mathrm{NH}_{2}\right), 6.89\left(\mathrm{~d}, \mathrm{~J}=8.4 \mathrm{~Hz}, 1 \mathrm{H}, \mathrm{CH}_{3}\right), 828$ 7.52-7.98 (m, 9H, Ph), $8.12(\mathrm{~d}$, $\mathrm{J}=8.4 \mathrm{~Hz}, 1 \mathrm{H}), 9.05\left(\mathrm{~d}, \mathrm{~J}=8.4 \mathrm{~Hz}, 1 \mathrm{H}, \mathrm{CH}_{2}\right), 9.19\left(\mathrm{~d}, \mathrm{~J}=8.4 \mathrm{~Hz}, 1 \mathrm{H}, \mathrm{CH}_{2}\right)$ ppm. UV-Vis $\left(\mathrm{CHCl}_{3}\right)$ : $\lambda_{\max }=271.21\left(\pi-\pi^{*}\right.$, Ar-H), $441.45\left(\pi-\pi^{*}, \mathrm{~N}=\mathrm{N}\right) \mathrm{nm}$.

1-(4-chlorophenylazo)-2-naphthol (Table 1, entry 2)

Orange red solid, FT-IR (KBr): $v_{\max }: 3130-3300,1598,1510,1438,1243,1091,825,749 \mathrm{~cm}^{-1}{ }^{1} \mathrm{H}$ NMR $\left(400 \mathrm{MHz}, \mathrm{CDCl}_{3}\right): \delta=6.9(\mathrm{~s}, 1 \mathrm{H}), 7.35-7.70(\mathrm{~m}, 9 \mathrm{H}), 9.53(\mathrm{~s}, 1 \mathrm{H}, \mathrm{OH}), 16.1(\mathrm{~s}, 1 \mathrm{H}, \mathrm{NH})$ ppm. UV-Vis $\left(\mathrm{CHCl}_{3}\right): \lambda_{\max }(\mathrm{nm})=360.80\left(\pi-\pi^{*}, \mathrm{Ar}-\mathrm{H}\right), 482.40\left(\pi-\pi^{*}, \mathrm{~N}=\mathrm{N}\right)$.

1-(2-(2, 4-dimethyl phenyl azo)-4, 6-dimethyl phenyl azo)-2-naphthol (Table 1, entry 3)

Dark red solid, mp 161-163 ${ }^{\circ} \mathrm{C}$, FT-IR (KBr): $v_{\max }: 3280-3570,3028,2977,1612,1497,1443$, 1383, 1206, $750 \mathrm{~cm}^{-1} .{ }^{1} \mathrm{H}$ NMR $\left(400 \mathrm{MHz}, \mathrm{CDCl}_{3}\right): \delta=2.36(\mathrm{~s}, 9 \mathrm{H}), 2.49(\mathrm{~s}, 3 \mathrm{H}), 6.84(\mathrm{~d}, \mathrm{~J}=9.6 \mathrm{~Hz}$, $1 \mathrm{H}), 7.08-7.21(\mathrm{~m}, 4 \mathrm{H}), 7.43(\mathrm{t}, \mathrm{J}=8 \mathrm{~Hz}, 1 \mathrm{H}), 7.61-7.76(\mathrm{~m}, 3 \mathrm{H}), 8.03(\mathrm{~s}, 1 \mathrm{H}), 8.56(\mathrm{~d}, \mathrm{~J}=7.6 \mathrm{~Hz}, 1 \mathrm{H})$, 
$9.51(\mathrm{~s}, \mathrm{OH}), 16.43(\mathrm{~s}, 1 \mathrm{H})$ ppm. UV-Vis $\left(\mathrm{CHCl}_{3}\right): \lambda \max =368.48\left(\pi-\pi^{*}, \mathrm{Ar}-\mathrm{H}\right), 487.52\left(\pi-\pi^{*}, \mathrm{~N}=\mathrm{N}\right)$ $\mathrm{nm}$.

12-(4-(4-hydroxyphenylazo)phenyl)-9, 9-dimethyl-8, 9, 10, 12-tetrahydrobenzo(a)xanthen-11-one (Table 1, entry 11)

Yellow solid, $\mathrm{mp} 263-265^{\circ} \mathrm{C}$. FT-IR (KBr): $v_{\max }$ : 3120-3350, 2954, 1628, 1616, 1590, 1503, 1466, 1439, 137, 1266, 1238, 1226, 1184, 1032, 838, 828, 814,744. ${ }^{1} \mathrm{H}$ NMR (500 MHz, $\left.\mathrm{CDCl}_{3}, \mathrm{DMSO}\right): \delta$ $=0.88(\mathrm{~s}, 3 \mathrm{H}), 1.05(\mathrm{~s}, 3 \mathrm{H}), 2.16(\mathrm{~d}, \mathrm{~J}=16.3 \mathrm{~Hz}, 1 \mathrm{H}), 2.24(\mathrm{~d}, \mathrm{~J}=16 \mathrm{~Hz}, 1 \mathrm{H}), 2.52(\mathrm{~s}, 2 \mathrm{H}), 5.67(\mathrm{~s}, 1 \mathrm{H})$, $6.82(\mathrm{~d}, \mathrm{~J}=8.2 \mathrm{~Hz}, 2 \mathrm{H}), 7.26-7.38(\mathrm{~m}, 6 \mathrm{H}), 7.57(\mathrm{~d}, \mathrm{~J}=7.7 \mathrm{~Hz}, 2 \mathrm{H}), 7.65(\mathrm{t}, \mathrm{J}=8.3 \mathrm{~Hz}, 2 \mathrm{H}), 7.71(\mathrm{t}$, $\mathrm{J}=8.2 \mathrm{~Hz}, 2 \mathrm{H}), 7.89(\mathrm{~d}, \mathrm{~J}=8.1 \mathrm{~Hz}, 1 \mathrm{H}) \mathrm{ppm}$. UV-Vis $\left(\mathrm{CHCl}_{3}\right): \lambda_{\max }(\mathrm{nm})=272.48\left(\pi-\pi^{*}, \operatorname{Ar}-\mathrm{H}\right)$, $353.12\left(\pi-\pi^{*}, \mathrm{~N}=\mathrm{N}\right)$.

12-(4-(2-hydroxynaphthylazo)phenyl)-9, 9-dimethyl-8,9,10,12-tetrahydrobenzo(a)xanthen-11-one (Table 1, entry 12)

Orange red solid, mp $123-126{ }^{\circ} \mathrm{C}$. FT-IR (KBr): v $v_{\max }: 3200-3535,3058,2956,2875,1652,1621$, 1596, 1467, 1502, 1459, 1372, 1225, 1180, 1026, 839, 813, $748 \mathrm{~cm}-1 .{ }^{1} \mathrm{H}$ NMR $\left(500 \mathrm{MHz}, \mathrm{CDCl}_{3}\right.$, DMSO): $\delta=1.02(\mathrm{~s}, 3 \mathrm{H}), 1.17(\mathrm{~s}, 3 \mathrm{H}), 2.30(\mathrm{~d}, \mathrm{~J}=16.3 \mathrm{~Hz}, 1 \mathrm{H}), 2.37(\mathrm{~d}, \mathrm{~J}=16.3 \mathrm{~Hz}, 1 \mathrm{H}), 2.63(\mathrm{~s}$, 2H), $5.80(\mathrm{~s}, 1 \mathrm{H}), 6.88(\mathrm{~d}, \mathrm{~J}=9.3 \mathrm{~Hz}, 1 \mathrm{H}), 7.35-7.47(\mathrm{~m}, 7 \mathrm{H}), 7.49(\mathrm{~d}, \mathrm{~J}=8.4 \mathrm{~Hz}, 2 \mathrm{H}), 7.60$ (t, J=6.4 Hz, $1 \mathrm{H}), 7.71(\mathrm{~d}, \mathrm{~J}=9.4 \mathrm{~Hz}, 2 \mathrm{H}), 7.80-7.84(\mathrm{~m}, 2 \mathrm{H}), 8.01(\mathrm{~d}, \mathrm{~J}=8.3 \mathrm{~Hz}, 1 \mathrm{H}), 8.52(\mathrm{~d}, \mathrm{~J}=8.1 \mathrm{~Hz}, 1 \mathrm{H}) \mathrm{ppm}$. UV-Vis $\left(\mathrm{CHCl}_{3}\right): \lambda_{\max }(\mathrm{nm})=269.92\left(\pi-\pi^{*}, \mathrm{Ar}-\mathrm{H}\right), 490.08\left(\pi-\pi^{*}, \mathrm{~N}=\mathrm{N}\right)$.

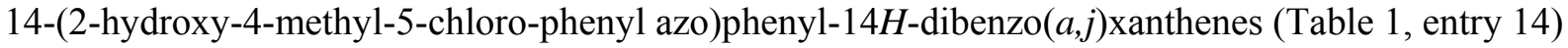

Orange red solid FT-IR: $v_{\max }($ ATR, neat $)=2900-3100,1593,1514,1478,1459,1431,1400,1247$, 1163, 810, 746, 1H NMR (400 MHz, $\left.\mathrm{CDCl}_{3}\right): \delta=2.31(\mathrm{~s}, 3 \mathrm{H}), 6.57(\mathrm{~s}, 1 \mathrm{H}), 6.60(\mathrm{~d}, \mathrm{~J}=8.8 \mathrm{~Hz}, 1 \mathrm{H})$, 6.71(s, $1 \mathrm{H}), 6.82(\mathrm{~s}, 1 \mathrm{H}), 7.17(\mathrm{~d}, \mathrm{~J}=8.8 \mathrm{~Hz}, 1 \mathrm{H}), 7.39(\mathrm{t}, \mathrm{J}=7.6 \mathrm{~Hz}, 2 \mathrm{H}), 7.51(\mathrm{~d}, \mathrm{~J}=8.8 \mathrm{~Hz}, 2 \mathrm{H}), 7.6(\mathrm{~m}$, $3 \mathrm{H}), 7.66(\mathrm{~m}, 1 \mathrm{H}), 7.77(\mathrm{~s}, 1 \mathrm{H}), 7.84(\mathrm{t}, \mathrm{J}=8.8 \mathrm{~Hz}, 4 \mathrm{H}), 8.37(\mathrm{~d}, \mathrm{~J}=8.8 \mathrm{~Hz}, 2 \mathrm{H}) \mathrm{ppm}$.

\section{References}

1 Karci, F.; Demircali, A.; Sener, I.; Tilki, T. (2006) Synthesis of 4-amino-1Hbenzo[4,5]imidazo[1,2-a]pyrimidin-2-one and its disperse azo dyes. Part 1: Phenylazo derivatives, Dyes and Pigments 71, 90-96.

2 Sharma, P.; Rane, N.; Gurram, V. K. (2004) Synthesis and QSAR studies of pyrimido[4,5d]pyrimidine- 2, 5-dione derivatives as potential antimicrobial agents, Bioorg. Med. Chem. Lett. $14,4185-4190$.

3 Sadegi, B.; Mirjalili, B. F.; Hashemi, M. M. (2008) $\mathrm{BF}_{3} \cdot \mathrm{SiO}_{2}$ : An efficient reagent system for the one-pot synthesis of 1,2,4,5-tetrasubstituted imidazoles, Tetrahedron Lett. 49, 2575-2577.

4 Mirjalili, B.F.; Bamoniri, A.; Akbari, A. (2008) $\mathrm{BF}_{3} \cdot \mathrm{SiO}_{2}$ : An efficient alternative for the synthesis of 14-aryl or alkyl-14H-dibenzo[a,j]xanthenes, Tetrahedron Lett. 49, 6454-6456.

5 Mirjalili, B. F.; Bamoniri, A.; Akbari, A. (2011) Nano- $\mathrm{BF}_{3} \cdot \mathrm{SiO}_{2}$ : a reusable and eco-friendly catalyst for synthesis of quinoxalines, Chem. Heterocycl. Comp., 47, 487-491.

6 Mirjalili, B. F.; Bamoniri, A.; Akbari, A. (2011) One-Pot Synthesis of 3,4-Dihydropyrimidin2(1H)-ones (Thiones) Promoted by Nano-BF $3 . \mathrm{SiO}_{2}$, J. Iran. Chem. Soc., 8, S135-S140.

7 Mirjalili, B. F.; Bamoniri, A.; Akbari, A. (2011) Nano- $\mathrm{BF}_{3} \cdot \mathrm{SiO}_{2}$ : Areusable and eco-friendly catalyst for thioacetalization and trans-thioacetalization reactions, Iranian J. Catal. 1, 87-92.

8 Pourjavadi, A., Mirjalili, B. F., Entezami, A. A.; Zohuriaan-Mehr, M. J. (2001), Novel azo containig polymethacrylates bearing spiroacetalmoeity and methylene spacers: synthesis and characterization, Eur. Poly. J. 37, 2111-2121. 